Essay review

\title{
Apparition, évolution et fin(?) du concept de schizophrénie
}

Marc Geiser

Bleuler E., Dementia Praecox ou groupe des psychoses schizophréniques, Paris, EPEL / GREC, 1993. [trad. de Dementia Praecox oder Gruppe der Schizophrenien, 1911.]

Boyle M., Schizophrenia. A scientific delusion?, Londres, Routledge, 1990.

Garrabé J., Histoire de la schizophrénie, Paris, Seghers, 1992.

Hoenig J., «Schizophrenia (clinical section)», in: Berrios G., Porter R. eds, A History of Clinical Psychiatry, Londres, Athlone,1995.

Howells J. G., The Concept of Schizophrenia: Historical perspective, Washington, American Psychiatric Press, 1991.

Turner T., «Schizophrenia (social section)», in: Berrios G., Porter R. eds, op. cit.

Comme le fait remarquer Trevor Turner, «le terme de schizophrénie, ses significations linguistiques, sociales et diagnostiques sont inextricablement liés au $\mathrm{XX}^{\mathrm{e}}$ siècle». C'est justement le sens de ce mot, l'évolution du concept de schizophrénie, que nous allons tenter de retracer ici à travers diverses contributions récentes consacrées à l'histoire de cette affection.

John Hoenig présente très succinctement les circonstances de la naissance du concept de schizophrénie, ainsi que ses développements ultérieurs à travers la trilogie Kraepelin-Bleuler-Schneider. L'actualité de la schizophrénie, du moins dans le monde francophone, c'est aussi la traduction en français par Alain Viallard du fameux texte d'Eugen Bleuler, Dementia Praecox oder Gruppe der Schizophrenien. La préface de cet ouvrage ${ }^{1}$ replace le Zurichois dans le contexte de la psychanalyse émergeante; le texte est suivi d'un essai de Jean Ey, datant de 1940, qui ne tarit pas d'éloges sur les conceptions «du maître de Zürich». L'histoire de la schizophrénie de Jean Garrabé est certai-

1 Rancher B., Zimra G., Rondepierre J.-P., Viallard A., «Bleuler, entre psychiatrie et psychanalyse?», in: Bleuler E., op. cit.

Marc Geiser, 2, rue des Voisins, 1205 Genève 
nement l'ouvrage le plus riche, mais l'on s'égare facilement dans cette surabondance d'anecdotes et de détails. Enfin, les deux derniers ouvrages étudiés présentent des points de vue diamétralement opposés: le plus ancien, celui de Mary Boyle,s'inscrit dans la tradition de l'antipsychiatrie; pour elle, la schizophrénie est une chimère qui repose sur des données erronées et une méthode impropre à l'observation des comportements humains. The Concept of Schizophrenia: historical perspectives, publié par les éditeurs du Diagnostic and Statistical Manual of Mental Disorders (ci-après DSM) propose des contributions d'un niveau très inégal comme nous le verrons.

\section{Préhistoire de la Schizophrenie}

La première contribution du livre édité par l'American Psychiatric Press adopte une approche qui suppose une invariance ontologique de la maladie. L'auteur, G. Roccatagliata, couvre la période s'étendant du VII siècle av. J.-C. au $\mathrm{XI}^{\mathrm{e}}$ siècle de notre ère. Sa conclusion illustre parfaitement sa méthode: «Il ne semble pas y avoir de différences notables entre la nosologie des troubles schizophréniques proposée par le DSM de l'American Psychiatric Association d'un côté et celle de la nosologie classique de l'autre.» A l'opposé de cette approche, M. Boyle tente de replacer la naissance du concept de schizophrénie dans le cadre plus général de l'évolution de la psychiatrie avant Emil Kraepelin en s'appuyant sur les grands spécialistes de la matière - Scull et Zilboorg, par exemple. Elle relève trois grands changements survenant entre le milieu du XVIII ${ }^{\mathrm{e}}$ siècle et le début du XX ${ }^{\mathrm{e}}$ : d'une part le développement des institutions chargées de «gérer» les aliénés, d'autre part la médicalisation de la folie et enfin, durant la seconde partie du XIX ${ }^{\mathrm{e}}$ siècle, l'apparition d'un système de classification.

Les autres historiens confinent la préhistoire de la schizophrénie au XIX siècle et à quelques personnages. Le terme de Dementia Praecox (l'ancien nom de la schizophrénie) est employé pour la première fois en 1852 par le français Augustin B. Morel. Il décrit ainsi l'état de jeunes aliénés tombant prématurément dans la démence. Un autre apport du psychiatre français est le concept étiologique de «dégénérescence».

Le concept de psychose unique avait été adopté par un courant important de la psychiatrie au XIX ${ }^{\mathrm{e}}$ siècle. Selon cette conception que l'on doit à Griesinger, il n'y aurait pas plusieurs maladies mentales, mais des formes différentes d'un seul et même processus inéluctable s'achevant par la démence. Selon B. Rancher et ses collègues, c'est contre cette idée que Ludwig Kahlbaum publie en 1863 son ouvrage Classification des maladies mentales. Il y 
propose de distinguer des entités spécifiques correspondant à autre chose qu'à des étapes successives vers la démence telles que la théorie de la psychose unique les considérait jusque là. Pour ce faire, il se base uniquement sur la clinique et l'évolution, s'inspirant, comme le suggère J. Garrabé, de l'école de la Salpêtrière.

Onze ans plus tard, Kahlbaum décrit ainsi la catatonie, un trouble caractérisé par une stupeur et des troubles moteurs. Ewald Hecker, l'un de ses élèves, isole en 1871 l'hébéphrénie. Cette affection touche principalement les adolescents et se manifeste par une détérioration progressive des fonctions psychiques; elle recouvre à peu près la Dementia Praecox de Morel.

Pour J. Hoenig, Morel fournit à Kraepelin le concept d'hérédité et le nom, tandis que Hecker et Kahlbaum lui offrent 2 groupes de diagnostics.

\section{Kraepelin}

C'est en 1883, alors qu'il enseigne la psychiatrie à Dorpat en Estonie, qu'Emil Kraepelin publie la première des huit éditions de son traité de psychiatrie. Cependant, c'est au cours de la décennie suivante que le psychiatre allemand, désormais professeur à Heidelberg, va bouleverser la psychiatrie en proposant de classer les psychoses selon la méthode clinique de Kahlbaum. Le critère évolutif est alors considéré comme fondamental: «C'est le passage de la conception symptomatique à la conception clinique...Partout l'importance des signes extérieurs de la maladie a dû reculer derrière les points de vue qui ressortent des conditions d'apparition, d'évolution et de terminaison» (Kraepelin, cité par J. Garrabé). Cette méthode basée sur la clinique lui permet de définir de véritables maladies, non pas seulement des syndromes.

Si pour tous les auteurs, l'histoire de la schizophrénie débute indiscutablement avec Kraepelin, en revanche, il subsiste un désaccord sur le texte dans lequel le psychiatre allemand jette les bases de la définition moderne du terme. Pour J. Hoenig et les auteurs de la préface de Bleuler, Kraepelin utilise pour la première fois le terme de Dementia Praecox dans la $4^{\mathrm{e}}$ édition de son traité (1893). Le diagnostic fait partie du groupe des «processus psychiques de dégénérescence» composé de trois entités: la démence précoce, correspondant à l'hébéphrénie de Hecker, la catatonie de Kahlbaum et la démence paranoïde; toutes trois sont caractérisées par une évolution déficitaire se terminant par un affaiblissement psychique. Pour M. Boyle, cette édition du traité de Kraepelin ne marque pas la naissance du concept dans le sens où il sera utilisé ultérieurement, mais le terme est ici employé par le psychiatre de Heidelberg dans la même acception que Morel une quarantaine d'années 
plus tôt. Pour l'historienne, tout comme pour J. Garrabé, c'est la $5^{\mathrm{e}}$ édition (1896) qui est le véritable point de départ de l'histoire de la schizophrénie, car pour ce dernier, le critère nosologique évolutif est retenu par Kraepelin pour la première fois dans ce texte. D'autres, comme U. H. Peters ${ }^{2}$, considèrent l'édition de 1899 (la $6^{\mathrm{e}}$ ) comme la genèse de cette histoire. En effet, c'est à cette date que l'ensemble du groupe des psychoses déficitaires est regroupé sous le terme de Dementia Praecox, tandis que l'hébéphrénie retrouve son ancienne signification. Un concept unique couvre désormais trois formes cliniques jusqu'alors considérées de manière distincte.

Lors des éditions ultérieures (1903-1904 pour la $7^{\text {e }}$ et 1909-1915 pour la $8^{\mathrm{e}}$ ), le concept connaît une grande extension: les trois entités cliniques sont divisées en 10 sous-types. En effet, Kraepelin reconnaît que la démence précoce se manifeste de façon très variée, d'où un certain malaise que J. Hoenig relève dans la huitième édition: «Il y a sans aucun doute une variété de formes récurrentes, mais les nombreuses transitions de l'une à l'autre rendent impossible des définitions précises et l'assignation univoque de chaque cas à un type particulier.» ${ }^{3}$ M. Boyle met systématiquement en évidence ces hésitations et nous apprend, par ailleurs, qu'entre la $6^{\mathrm{e}}$ et la $8^{\mathrm{e}}$ édition, on assiste à une véritable inflation des comportements tenus pour être des symptômes de la Dementia Praecox: en 1896,37 pages suffisaient, tandis que la $8^{\mathrm{e}}$ édition comporte pas moins de 356 pages sur la question!

Déjà en 1899, les conceptions kraepeliniennes ont fait l'objet de différentes critiques dont nous fait part J. Garrabé. Serbski, notamment «s'étonne que des états dont la clinique est aussi différente que l'hébéphrénie avec ses troubles formels du langage, la catatonie avec ses spectaculaires manifestations psychomotrices et les délires paranoïdes avec leur intense activité hallucinatoire puissent n'être que de simples formes d'une seule maladie». On reproche aussi au psychiatre allemand le fait que seul le stade final soit retenu comme critère nosologique, car en effet, de cette manière, la maladie ne peut être diagnostiquée que rétrospectivement. L'évolution terminale pose un autre problème, car, de l'aveu même de Kraepelin, l'affaiblissement psychique ne se produit pas toujours, d'où les sarcasmes de Serbski sur «la démence sans démence». Enfin, il est aussi reproché à Kraepelin d'avoir abandonné l'analyse psychologique des symptômes: il les considère comme des signes objectifs de la maladie sans chercher à les interpréter.

En 1917, von Economo a décrit les séquelles d'une maladie neurologique (l'encéphalite léthargique) présentant d'étonnantes similitudes avec la schi-

2 Peters U. H., «The German Classical Concept of Schizophrenia», in: Howells J. G., op. cit., p. 62.

3 Kraepelin, $8^{e}$ édition, cité par J. Hoenig. 
zophrénie. J. Garrabé mentionne cette «nouvelle maladie» apparue en 1916 sous la forme d'une épidémie. M. Boyle relève, de son côté, que certains chercheurs ont trouvé des traces de cette affection aussi au XVIII et à la fin du $\mathrm{XIX}^{\mathrm{e}}$ siècle. Par conséquent, il est fort possible que des patients de Kraepelin aient été atteints de cette maladie. Cette hypothèse expliquerait que l'on ne rencontre plus de nos jours certains symptômes ou des cas graves et de longue durée, décrits au tournant du siècle.

\section{Bleuler}

En 1911, l'histoire de la Dementia Praecox va connaître une importante inflexion. Cette année-là, Eugen Bleuler, alors directeur de la clinique du Burghölzli, publie son Dementia Praecox oder Gruppe der Schizophrenien. Comme le titre l'indique, le psychiatre zurichois crée un néologisme pour remplacer le terme proposé par Kraepelin. Il le fait d'abord pour des raisons presque poétiques, comme il l'explique lui-même: «Nous ne pouvons malheureusement nous soustraire à la désagréable tâche de forger un nouveau terme pour ce groupe nosologique. Celui qui est en usage jusqu'à présent est trop peu maniable. On ne peut s'en servir que pour dénommer la maladie, mais non les malades, et l'on ne peut former d'adjectif qui puisse qualifier les caractéristiques propres à cette maladie (...). Il serait fâcheux de rédiger un diagnostic différentiel détaillé sans disposer d'un tel terme, et plus fâcheux encore de le lire.» Mais des impératifs théoriques sont aussi à l'origine de cette métamorphose sémantique. D'une part, le terme Dementia Praecox ne semble plus approprié: «l'ancien mot a été créé à une époque où tant le concept de démence que celui de précocité pouvaient s'appliquer à presque tous les cas qu'on y incluait. Il n'est plus adapté à l'étendue actuelle du concept nosologique, car il ne s'agit ni uniquement de malades que l'on puisse qualifier de déments, ni exclusivement d'abêtissements précoces.» D'autre part, le nouveau terme est construit pour désigner la caractéristique principale de cette affection: «J'appelle la démence précoce schizophrénie parce que, comme j'espère le montrer, la scission des fonctions psychiques les plus diverses est l'un de ses caractères les plus importants. Pour des raisons de commodité, j'emploie ce mot au singulier, bien que ce groupe comprenne vraisemblablement plusieurs maladies.» ${ }^{4}$

Les auteurs de la préface présentent de manière très claire sa théorie. Le psychiatre suisse organise les symptômes en deux groupes: «Les symptômes

4 Bleuler E., op. cit., pp. 43-44. 
primaires seraient l'indice d'un processus morbide, alors que les secondaires pourraient être tenus pour la réaction de l'esprit malade aux événements internes ou externes.» ${ }^{5}$ Cependant, ces deux groupes n'ont pas le même poids: «le facteur causal organique supposé de la maladie est le primum movens», mais il produit des signes primaires peu nombreux. Au contraire, bien que les signes secondaires d'origine psychique constituent l'essentiel de la symptomatơlogie, ils ne représentent pas un facteur causal de la maladie. Parallèlement à cette séparation théorique (primaires, secondaires), Bleuler fait une distinction clinique entre les symptômes fondamentaux que l'on trouve en permanence chez tous les schizophrènes et les symptômes accessoires que l'on ne rencontre pas forcément.

Le symptôme primaire fondamental est le relâchement primaire des associations qui serait la cause d'un affaiblissement des fonctions logiques au profit des complexes affectifs; cette subordination aux complexes briserait l'unité de la personnalité. C'est cette dislocation que Bleuler nomme Spaltung (scission, ou le «schisis» de schizophrénie).

Bien que Bleuler n'ait jamais prétendu rompre avec les conceptions de Kraepelin, c'est pourtant les différences entre ces deux psychiatres qui frappent le plus les historiens. Tandis que Kraepelin a une approche empirique basée sur l'observation des symptômes, Bleuler suit une théorie, en structurant les symptômes (primaires et secondaires). J. Hoenig remarque au passage fort justement que ce partage semble erroné au vu des résultats que produisent les neuroleptiques. En effet, contrairement à ce que laissait supposer la théorie de Bleuler, ce sont les symptômes psychogéniques qui réagissent à ces médicaments alors que les symptômes primaires produits par un processus organique ne sont que très peu affectés.

Comme le soulignent J. Garrabé et J. Hoenig, la rupture avec les conceptions kraepeliniennes réside aussi et surtout dans l'application de l'analyse psychologique à l'étude de la démence précoce: les symptômes ne sont pas seulement observés, mais interprétés en référence aux complexes. Bleuler, qui comptait parmi ses assistants K. Abraham jusqu'en 1907 et C.-G. Jung jusqu'en 1911, s'intéresse activement à la psychanalyse, alors en cours d'élaboration. Il est fasciné par Freud au point de rapprocher ses descriptions de l'autisme (dont souffrent certains schizophrènes) du concept d'autoérotisme freudien, alors qu'en fait, comme le démontrent les auteurs de la préface, l'autisme bleulérien recouvre l'autoérotisme défini par Jung comme un monde intérieur, un espace imaginaire où les complexes jouent leur jeu autoérotique. Bleuler renonce pourtant, en 1926, après avoir 
adopté une position organiciste, à la psychothérapie pour soigner la schizophrénie.

Les auteurs de la préface estiment que Bleuler a parcouru en sens inverse le chemin fait par Kahlbaum, Hecker et Kraepelin. En effet, «l'extension qu'il donne au concept de démence précoce, en y incluant toutes les hypocondries, hystéries, manies, mélancolies atypiques, etc., en fait une sorte de fourre-tout. (...) Ainsi les schizophrénies deviennent-elles pratiquement la psychose unique» ${ }^{6}$. En fait, font-ils remarquer, elles s'étendent même au-delà de la pathologie mentale déclarée avec la schizophrénie latente, une forme asymptomatique décelable uniquement grâce à une observation patiente. Cette catégorie a permis d'inclure beaucoup de personnes dont la seule maladie était une mauvaise intégration sociale.

\section{La schizophrénie après Kraepelin et Bleuer}

Après Kraepelin et Bleuler, un troisième homme semble incontournable dans l'histoire de la schizophrénie. Il s'agit de Kurt Schneider, titulaire depuis 1945 de la chaire de psychiatrie de Heidelberg. Il s'inscrit dans la tradition descriptive de son illustre prédécesseur, Kraepelin, en proposant des symptômes de $1^{\text {er }}$ rang permettant de diagnostiquer la schizophrénie et des symptômes de $2^{\mathrm{e}}$ rang. Cependant, la distinction, bien que purement descriptive, n'est pas très claire. M. Boyle relève les contradictions de Schneider qui d'un côté estime que les symptômes de $1^{\text {er }}$ rang sont très importants pour poser un diagnostic, mais qui d'un autre côté affirme que les cliniciens sont parfois obligés de se baser sur les symptômes de $2^{\mathrm{e}}$ rang. Charles P. Peters va dans la même direction en rapportant les résultats d'une étude révélant que moins d'un quart des patients souffrant de schizophrénie ou de psychose maniaco-dépressive présentaient des symptômes de $1^{\text {er }} \mathrm{rang}^{7}$. De son côté, J. Garrabé regrette que la clinique de Schneider soit une clinique du moment et que l'approche diachronique de Kraepelin ait été abandonnée. Néanmoins, les symptômes dits de premier rang serviront de critères de base dans les systèmes internationaux de diagnostic.

Immédiatement après la seconde guerre mondiale, les premières tentatives de mettre au point un système international de diagnostic voient le jour. Dans la $6^{e}$ édition de l'ICD (The International Statistical Classification of Diseases, Injuries and Causes of Death), publiée en 1948 par l'OMS, la

6 ibid., p. 14.

7 Peters C. P., «Concepts of Schizophrenia After Kraepelin and Bleuler», in: Howells J. G., op. cit., p. 99. 
schizophrénie apparait dans le chapitre des «Troubles schizophréniques ou Dementia Praecox». Dans la 9e édition de ce manuel parue en 1979, la définition des psychoses schizophréniques est axée sur quatre types de symptômes touchant la personnalité, la pensée (correspondant à peu près aux symptômes de $1^{\text {er }}$ rang de Schneider), l'activité délirante et l'affectivité. De leur côté, les Américains ont mis sur pied leur propre système: le Diagnostic and Statistical Manual of Mental Disorders publié pour la première fois en 1952. Le DSM-III publié quant à lui en 1980, bouleverse, selon J. Garrabé, de manière révolutionnaire la taxinomie psychiatrique. Les termes de «psychose» et «névrose» ont disparu, toutes les références à une théorie ou à un auteur en particulier sont évitées. Le terme même de schizophrénie disparaît au profit de celui de «techniquement adéquat» de «troubles schizophréniques» (le terme «Schizophrenia» resurgit dans le DMS-III R paru en 1987). Pour J. Garrabé, la validité du concept repose sur 3 critères: 1 . une différence de réponse aux traitements biologiques, 2 . des aspects familiaux particuliers, 3 . une tendance à survenir chez l'adulte jeune, avec récurrence et détérioration du fonctionnement social et professionnel. L'historien et psychiatre français relève que le critère kraepelinien d'évolution déficitaire est abandonné et que, par contre, la notion bleulérienne de schizophrénie comme groupe d'affections est consacrée. Il reste de manière générale d'ailleurs assez critique à l'égard du DSM-III qu'il accuse d'escamoter la schizophrénie. T. Turner, rappelant qu'une étude commanditée par l'OMS en 1973 avait mis en évidence que les psychiatres américains utilisaient le concept de schizophrénie dans un sens beaucoup plus large qu'ailleurs, estime que le DSM-III a réduit en revanche les limites de la définition pour ces derniers.

Bien que le terme de schizophrénie ait été formé par Bleuler pour permettre de dénommer le malade, le schizophrène, ou du moins le présumé schizophrène, est totalement absent des contributions passées en revue. Aucun auteur n'accorde la moindre place au patient et à sa façon de s'exprimer, au sens large du terme. La scène où jouent le psychiatre et le patient, n'est pas évoquée, ni à travers des témoignages, ni même à travers les archives des médecins. Certes, T. Turner dévoile de façon impressionniste la floraison du terme de «schizophrénie» au cours du siècle écoulé et mentionne, à l'occasion, des écrits de personnes diagnostiquées schizophrènes, mais sans pour autant approfondir ces témoignages ou les mettre en perspective.

Il semble, en fait, que ces historiens, au demeurant psychiatres pour la plupart, s'intéressent essentiellement aux «grands psychiatres» et à leurs textes majeurs en présentant le tout de manière chronologique, sans véritablement prêter attention aux égarements de la psychiatrie. Cependant, s'ils ne peu- 
vent contourner ces errances (comme dans le cas de l'utilisation abusive du diagnostic de schizophrénie latente dans les pays totalitaires), ils préfèrent mettre la faute sur le régime politique plutôt que de s'interroger sur les conditions qui ont permis l'apparition de tels diagnostics, car rappelons-le, ce n'est autre que Bleuler qui est à l'origine de cette conception très large de la maladie mentale. Au total, il apparait que ces psychiatres-historiens plongent dans le passé pour tenter de dissiper leurs doutes. Cependant, au vu de leurs conclusions, bien souvent pessimistes, ils n'y parviennent pas vraiment.

Même si l'historienne et psychologue M. Boyle n'examine que les textes de Kraepelin, Bleuler et Schneider, elle adopte pourtant une approche bien plus problématisée que les autres historiens. En effet, elle tente de démontrer que la méthode de ces précurseurs n'était pas scientifique. On peut tout de même lui reprocher de trop détacher les contributions de ces psychiatres du contexte dans lequel ces derniers évoluaient et de laisser transparaître derrière ses critiques des préoccupations bien plus actuelles. Pour T. Turner, en effet, de telles attaques reflètent le désir des psychologues de jouer un rôle plus dominant dans le traitement des personnes souffrant de maladie mentale.

A l'issue de ce parcours, nous laisserons la parole à B. Rancher et à ses collègues qui, nous semble-t-il, résument parfaitement bien la trajectoire du concept de schizophrénie et l'état actuel de la psychiatrie: «Après avoir été néanmoins considérée tout au long du $\mathrm{XX}^{\mathrm{e}}$ siècle comme la figure emblématique de la folie sans pour autant parvenir à en constituer le paradigme, la schizophrénie n'est-elle pas en passe de connaître depuis les années 1980, une remise en cause radicale par la mise en place du DSM-III qui réduit les psychoses et les névroses à n'être que des ‘Troubles〉, des 〈disorders〉? Cette dissolution de la nosographie en des ‘Troubles〉 où l'on pourrait être tenté de voir un équivalent des 〈tableaux d'état〉 antérieurs à l'édifice kraepelinien est-elle l'aveu d'un anéantissement de la clinique psychiatrique?» ${ }^{8}$

8 Rancher B., Zimra G., Rondepierre J.-P., Viallard A., «Bleuler, entre psychiatrie et psychanalyse?», in: Bleuler E., op. cit., p. 35. 